\title{
Cooperative Full-Duplex Physical and MAC Layer Design in Asynchronous Cognitive Networks
}

\author{
Teddy Febrianto, Jiancao Hou, and Mohammad Shikh-Bahaei \\ Centre for Telecommunications Research, King's College London, London WC2R 2LS, UK \\ Correspondence should be addressed to Teddy Febrianto; teddy.febrianto@kcl.ac.uk
}

Received 26 May 2017; Revised 29 August 2017; Accepted 17 September 2017; Published 19 November 2017

Academic Editor: Gonzalo Vazquez-Vilar

Copyright (C) 2017 Teddy Febrianto et al. This is an open access article distributed under the Creative Commons Attribution License, which permits unrestricted use, distribution, and reproduction in any medium, provided the original work is properly cited.

\begin{abstract}
In asynchronous cognitive networks (CNs), where there is no synchronization between primary users (PUs) and secondary users (SUs), spectrum sensing becomes a challenging task. By combining cooperative spectrum sensing and full-duplex (FD) communications in asynchronous CNs, this paper demonstrates improvements in terms of the average throughput of both PUs and SUs for particular transmission schemes. The average throughputs are derived for SUs and PUs under different FD schemes, levels of residual self-interference, and number of cooperative SUs. In particular, we consider two types of FD schemes, namely, FD transmit-sense-reception (FDr) and FD transmit-sense (FDs). FDr allows SUs to transmit and receive data simultaneously, whereas, in FDs, the SUs continuously sense the channel during the transmission time. This paper shows the respective trade-offs and obtains the optimal scheme based on cooperative FD spectrum sensing. In addition, SUs' average throughput is analyzed under different primary channel utilization and multichannel sensing schemes. Finally, new FD MAC protocol design is proposed and analyzed for FD cooperative spectrum sensing. We found optimum parameters for our proposed MAC protocol to achieve higher average throughput in certain applications.
\end{abstract}

\section{Introduction}

Cognitive networks (CNs), which can dramatically improve spectrum efficiency using dynamic spectrum access (DSA) technology, is a promising solution for the spectrum scarcity problem [1-3]. CNs allow the cognitive devices or secondary users (SUs) to use licensed or primary users' (PUs) frequency spectrum in an opportunistic way while guaranteeing the quality of both systems.

The majority of research works in this area have studied these problems in synchronous conditions, where PUs and SUs are time slotted and synchronized. However, in realistic scenarios, SUs have no information about the PUs' signals, which results in operations in asynchronous mode. Jiang et al. have studied some key issues for asynchronous CNs in [4].

Asynchronous cooperative spectrum sensing has been proposed in $[5,6]$ which shows improvement in the average throughput of SUs. In-band full-duplex (FD) communication [7] is also a promising technology that can improve the performance of CNs. FD spectrum sensing has been proposed in [8] which allows SUs sense and transmit data at the same time.

In shared-spectrum full-duplex networking, it is common for the FD transceivers to operate in the transmitsense mode, that is, to transmit and sense simultaneously and in the same frequency band $[9,10]$. In this mode no data is received during the data transmission period, in contrast with standard noncognitive full-duplex scenarios. It has been shown that operating in transmit-sense mode can reduce the outage probability of the primary network significantly, compared to the conventional listen-before-talk scheme (i.e., compared to the cognitive scenario where halfduplex transmission is performed following a short sensing period) [11].

An alternative approach to full-duplex networking in CNs is to combine sensing with data transmission and reception $[12,13]$. Exploiting full-duplex communication capability of the transceivers, data transmission takes place simultaneously with data reception in this scheme, as it does with standard full-duplex networks. However, to allow spectrum 
sharing with the primary network, the reception process is divided in time so that sensing and data receiving can take place in different time slots, while data transmission continues over the entire time period, that is, using time division duplex (TDD) in reception.

The advantage of transmit-sense mode approach is that it allows continuous sensing which can, in turn, improve the probability of detecting returning primary users. This improvement in the likelihood of detection is also a result of more advanced learning algorithms that can be implemented in continuous sensing. This is in contrast with data transmit-sense-reception mode, where sensing takes place in short periods of time; that is, data reception and sensing are scheduled according to a time-division-duplex scheme. Intermittent sensing in this mode does not allow the employment of advanced and reliable learning algorithms, which implies higher missed detection probability, compared to the transmit-sense mode. On the other hand, operation in transmit-sense-reception mode improves the secondary users' throughput at the cost of deteriorating primary network's performance.

The authors in $[12,13]$ have introduced adaptive fullduplex transmit-sense (FDs) and full-duplex transmitreceive-sense (FDr) to improve the performance of the spectrum-sharing mechanism. These two works use energybased sensing and waveform-based sensing, respectively. However, both of these works have not considered cooperative spectrum sensing in order to further improve the spectrum sensing accuracy. On the other hand, the effect on the primary network can be alleviated by deploying cooperative sensing. Cooperation among the secondary users in sensing the licensed channel can improve the quality of detecting the activity of primary users in the licensed spectrum. By combining cooperative sensing and full-duplex communication features, full-duplex cooperative spectrum sensing mechanism is analyzed to improve the average throughput of SUs, while guaranteeing PUs' quality and throughput. This solution has been proposed in [14] only for time-slotted PUs and in [15] for cooperative acknowledgement. The recent work in [16] has analyzed SUs' performance in non-timeslotted case, without looking into the imposed effect on PUs.

Motived by the above observation, the contributions of this paper can be summarized as follows. The throughput of both secondary and primary users is derived under FDr and FDs schemes as functions of residual self-interference (SI) and the number of cooperating users in spectrum sensing. Furthermore, the results are extended for different primary channel utilization. Unlike our previous work in [17], in this paper, we introduce the full-duplex cooperative multichannel based FDr and FDs sensing schemes and also find the minimum number of cooperating secondary users required in FDr scheme so that the achievable throughput for the primary users is very close to that in the FDs scheme. In the literature, the full-duplex cooperative multichannel scenarios have also been considered [8, 18, 19]. However, these works assume SUs perform the standard p-persistent carrier sense multiple access (CSMA) mechanism for contention resolution on the selected channel. Such distributed mechanism can effectively avoid the data crash and improve

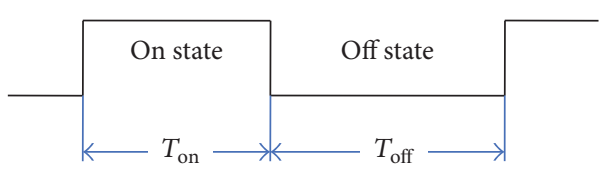

FIGURE 1: On-off process channel model.

the spectrum efficiency of the network. On the other hand, by comparing with the cooperative multichannel allocation mechanism which will be considered in this paper, the distributed version has to be limited to a "small" network. Based on the cooperative SUs design, in this paper, we also propose a MAC layer protocol design and analyze the average throughputs of SUs and PUs when utilizing TV white space as primary channels. The results show that, by increasing the number of sensed channels, the average throughput of proposed FD schemes may not always be improved, especially for FDr scheme. This is because the duration of sensing is considered as nontransmitting time, which reduce the time for data transmission.

The rest of this paper is organized as follows. The system model and throughput analysis are presented in Section 2. Spectrum sensing and self-interference effect are analyzed in Section 3. Formulation of secondary and primary average throughput for the cooperative full-duplex sensing scenario is in Sections 4 and 5, respectively. The analysis for the average throughput optimization and its corresponding numerical results are provided in Section 6. A new MAC protocol design is proposed and evaluated in Section 7. Finally, conclusions are drawn in Section 8.

\section{System Model}

As discussed in abstract and introduction sections, the asynchronous cognitive networks are defined that only the primary and secondary networks are not synchronized, but within the secondary network, the secondary users will be assumed to be synchronized. Moreover, the primary users in this case may or may not be synchronized. In addition, we also assume that SUs always have packets to "receive" as $[9,20]$, and this assumption is meaningful if the number of SUs is directly linked to the traffic demand in MAC protocol design.

2.1. Primary Users. One pair of PUs is considered to communicate in half-duplex (HD) mode over $W$ spectrum bands, where the bands are not overlapped and with the same spectrum utilization as $\beta$. PUs' channel is modeled as an onoff process as [21], which is shown in Figure 1. The channel is considered in on state when it is occupied by PUs. On the other hand, off state means there is an available spectrum that can be exploited by SUs. The length of the on state $\left(T_{\text {on }}\right)$ and off state $\left(T_{\text {off }}\right)$ follows the exponential distribution with averages of $\rho_{\text {on }}$ and $\rho_{\text {off }}$, respectively. In this case, the primary channel utilization $\beta$ can be calculated as

$$
\beta=\frac{\rho_{\text {on }}}{\rho_{\text {off }}+\rho_{\text {on }}} .
$$




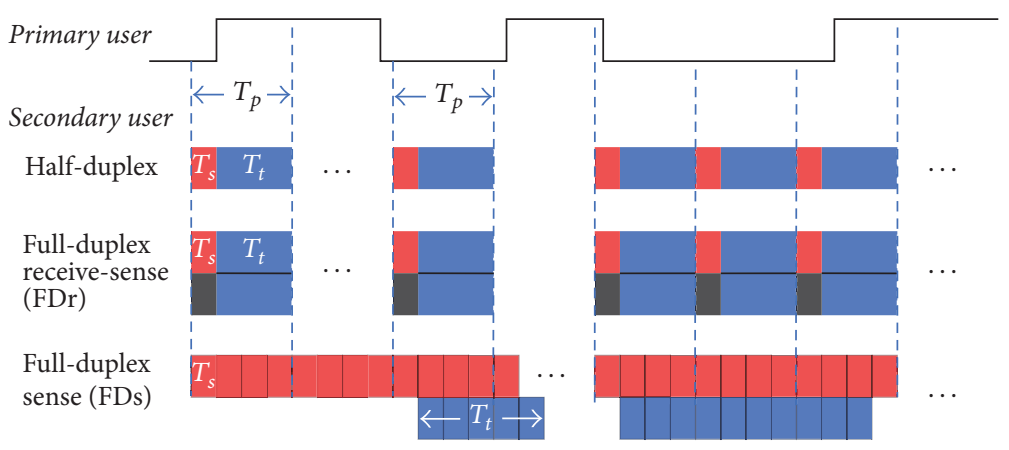

FIGURE 2: Secondary users transmission, reception, and sensing schemes.

2.2. Secondary Users. We consider that there are $M$ SUs that cooperate in sensing the PUs' spectrum bands. Among these $M$ cooperating SUs, there is one coordinator node which decides whether the primary channel is available for SUs. The coordinator node will collect the sensed information from $M$ collaborating SUs [5] and use it for "soft decision" [22]. If, during the SUs' transmission, the coordinator node realizes the PU's return to the channel, it would immediately inform the active SUs to stop opportunistic transmission in the primary band. Spectrum allocation by the coordinator is not studied in this paper, and the spectrum allocation scheme will be considered to be independent of the asynchronous spectrum sensing process.

As shown in Figure 2, we consider two full-duplex sensing mechanisms for SUs to use the available primary users' channels opportunistically. In addition, we also include the half-duplex (HD) mechanisms for comparison. Specifically, for the $\mathrm{HD}$ communication sensing process, SUs' frame is divided into two intervals, that is, sensing $\left(T_{s}\right)$ and transmitting $\left(T_{t}\right)$, with sampling rate of $\omega_{s}$. Energy-based sensing is applied for the sensing process of SUs. $T_{p}=T_{s}+T_{t}$ is defined as sensing period. In the second scheme, SUs use fullduplex communication to sense, transmit, and receive data within the same frame time $T_{p}$. This scheme is referred to as full-duplex transmit-receive-sense (FDr), and it allows SUs to receive data during the data transmitting process. The third scheme is full-duplex transmit-sense (FDs), where SUs sense the primary channel continuously during data transmission.

2.3. Average Throughputs. We assume that each SU can sense $v(\leq W)$ channels over $T_{p}$ seconds, where each channel is sensed for $T_{\text {so }}$ seconds. In this case, we have

$$
T_{s}=V \cdot T_{\text {so }} \text {. }
$$

In addition, $L$ is defined as the average number of sensed idle channels by $M$ SUs, which can be derived as [6]

$$
L=\sum_{v=0}^{W} v \cdot P_{v}(v),
$$

where $P_{v}(v)$ is the probability that $v$ idle channels can be sensed by $M$ SUs, and by considering (1), we have [6]

$$
P_{v}(v)=(L v) \beta^{L-v}(1-\beta)^{v} .
$$

Therefore, SU's average throughput with $v$ sensible channels can be expressed as

$$
\tau_{s, \text { scheme }}^{(v)}=\frac{L \cdot \tau_{s, \text { scheme }}}{W},
$$

where $\tau_{s, \text { scheme }}$ is SU's average throughput per channel for a specific sensing scheme, for example, HD, FDr or FDs. On the other hand, PU's average throughput (i.e., $\tau_{p \text {,scheme }}^{(v)}$ ) with multiple channels will have the same behaviour as for the single channel case (i.e., $\tau_{p \text {,scheme }}$ ) since all the channels are independently distributed. From following sections, we will mathematically derive the average throughputs per channel for both SU and PU in detail.

\section{Spectrum Sensing and Self Interference Effect}

The two fundamental measures to be evaluated in spectrum sensing are the detection probability $\left(P_{d}\right)$ and the false alarm probability $\left(P_{f}\right) . P_{d}$ is the probability that SUs can detect a busy channel when PUs do use the channel. $P_{f}$ is the probability that SUs falsely detect a busy channel whereas there is actually no PU activities.

Residual self-interference (SI) in full-duplex communication affects the detection probability and the probability of false alarms in sensing the activity of primary users. An energy detection technique is widely deployed for detecting the primary users' activity in the shared spectrum. In fullduplex sensing, that is, simultaneous data transmission and spectrum sensing in the same frequency band, the energy of the residual SI, as a result of imperfect SI cancellation, may be mistaken for primary users' signal. This, in turn, will increase the false alarm probability and reduce the secondary users' throughput. Waveform-based sensing is an alternative sensing method that can alleviate this problem in full-duplex scenarios. In this approach sensing the primary signals is carried out by correlating the received samples with known pattern samples.

Another alternative to energy detection is cyclostationary feature detection, which is based on the estimation of the Fourier spectrum cyclic density and can detect weak signals from primary users by only exploiting the cyclostationarity property of communication signals. However, this approach 
is rather complex for implementation. A different approach in detecting primary users' signals is based on tracking the primary users by employing smart antennas and avoiding spatial interference with their signals through transmit beamforming. Cooperative sensing can improve the probability of detecting primary signals, at the cost of higher computation and networking complexities. Using full-duplex radios, transmission and reception of data can be implemented simultaneously for further increase in the secondary user throughput. In this paper, cooperative energy-detectionbased sensing is considered in the analysis.

Hypotheses $H_{0}$ and $H_{1}$ correspond to the cases where primary channel is in the off state and on state, respectively. Under the $H_{0}$ and $H_{1}$ conditions, the SUs' received signals at time instant $n\left(r_{m}[n]\right)$ are, respectively, given by

$$
\begin{aligned}
& H_{0}: r_{m}[n]=x_{m}[n]+u_{m}[n], \\
& H_{1}: r_{m}[n]=x_{m}[n]+s_{m}[n]+u_{m}[n],
\end{aligned}
$$

where $n$ refers to the $n$th sample and subscript $m$ denotes the $m$ th SU. $x_{m}$ is the self-interference of $m$ th SU and $s_{m}$ is the signal transmitted by PU and received at the $m$ th SU. The background noise which is assumed as circular symmetric complex Gaussian is denoted by $u_{m}$. The mean of $u_{m}$ is zero, and the variance is $\sigma^{2}$.

The overall energy statistic of primary channel received at the coordinator $\mathrm{SU}(R)$ is given by [5]

$$
R=\frac{1}{M \omega_{s} T_{s}} \sum_{m=1}^{M} \sum_{n=1}^{\omega_{s} T_{s}}\left|r_{m}[n]\right|^{2}
$$

In addition, $P_{d}$ and $P_{f}$ are given by [8]

$$
\begin{aligned}
P_{d} & =\operatorname{Pr}\left[R \geq \varepsilon_{\mathrm{th}} \mid H_{1}\right] \\
& =Q\left(\frac{\varepsilon_{\mathrm{th}} / \sigma^{2}-((U-a) / U) \kappa \mathrm{SNR}_{s, p}-1}{\sqrt{\left((U-a) / U^{2}\right)\left(\kappa \mathrm{SNR}_{s, p}+1\right)^{2}+a / U^{2}}}\right), \\
P_{f} & =\operatorname{Pr}\left[R \geq \varepsilon_{\mathrm{th}} \mid H_{0}\right] \\
& =Q\left(\frac{\varepsilon_{\mathrm{th}} / \sigma^{2}-(d / U) \kappa \mathrm{SNR}_{s, p}-1}{\sqrt{\left(d / U^{2}\right)\left(\kappa \mathrm{SNR}_{s, p}+1\right)^{2}+(U-d) / U^{2}}}\right),
\end{aligned}
$$

$$
P_{f}=Q\left(\frac{\varepsilon_{\mathrm{th}} / \sigma^{2}-((d-\lfloor d / U\rfloor) / U) \kappa \mathrm{SNR}_{s, p}-1}{\sqrt{\left((d-\lfloor d / U\rfloor) / U^{2}\right)\left(\kappa \mathrm{SNR}_{s, p}+1\right)^{2}+(U-d+\lfloor d / U\rfloor) / U^{2}}}\right),
$$

where $\varepsilon_{\mathrm{th}}$ is the energy detection threshold, $U$ is the number of primary samples during the sensing period, $a$ is the number of samples during off state before primary's return to an active $(\mathrm{ON})$ state, and $d$ is the number of samples during in which the primary is at on state before becoming inactive. $\mathrm{SNR}_{x, y}$ is the signal-to-noise ratio at receiver $x$ due to the signal transmitted by transmitter $y . x, y \in\{p, s\}$, where $p$ and $s$, respectively, refer to a primary and secondary user. $\kappa(0<\kappa \leq$ 1) represents the self-interference mitigation coefficient $[8$, 23]. If $\kappa$ is high, this means that self-interference is mitigated well. On the other hand, low values of $\kappa$ imply that self interference at the receiver is high. $Q(\cdot)$ is the complementary distribution function of standard Gaussian, which is defined by

$$
Q(x)=\frac{1}{\sqrt{2 \pi}} \int_{x}^{\infty} e^{\left(-t^{2} / 2\right)} d t .
$$

Consider multichannel sensing scenarios, where PUs may change their states (i.e., on or off) during SUs' sensing period. In this case, there are four possible cases when we calculate $P_{d}$ and $P_{f}$ in order to obtain the average throughputs.

Case 1. PU is not active during the SUs' sensing period. In this case, $P_{f}$ which is used for SUs' achievable throughput calculation can be expressed as

$$
P_{f}=Q\left(\left(\frac{\varepsilon_{\mathrm{th}}}{\sigma^{2}}-1\right) \sqrt{M \omega_{s} T_{s}}\right)
$$

where this equation is derived by setting $d=0$ and $U=$ $M \omega_{s} T_{s}$.

Case 2. PU is always active during the SUs' sensing period. In this case, $P_{d}$ which is used for PU's achievable throughput calculation can be expressed as

$$
P_{d}=Q\left(\left(\frac{\varepsilon_{\mathrm{th}}}{\sigma^{2}}-\kappa \mathrm{SNR}_{s, p}-1\right) \sqrt{\frac{M \omega_{s} T_{s}}{\left(\kappa \mathrm{SNR}_{s, p}+1\right)^{2}}}\right),
$$

where, here, we set up $a=0$ and $U=M \omega_{s} T_{s}$.

Case 3. PU is firstly active until the $d$-th sample and then not active for the rest. In this case, $P_{d}$, which is used to calculate the PU's achievable throughput, is derived from (11). For calculating the SUs' throughput, apart from $P_{f}$ in (10), they still need to take the following $P_{f}$ into account, which is given by where $U=M \omega_{s} T_{s}$ and $\lfloor a\rfloor$ represents the maximum integer that is smaller than $a$.
Case 4. PU is firstly not active until the $a$-th sample and then active for the rest. In this case, $P_{f}$ which is used to 
calculate the SUs' average throughput is derived from (10). For calculating the PU's average throughput, apart from $P_{d}$ in (11), they also need to take the following $P_{d}$ into account, which is given by

$$
P_{d}=Q\left(\frac{\varepsilon_{\mathrm{th}} / \sigma^{2}-((U-a+\lfloor a / U\rfloor) / U) \kappa \mathrm{SNR}_{s, p}-1}{\sqrt{\left((U-a+\lfloor a / U\rfloor) / U^{2}\right)\left(\kappa \mathrm{SNR}_{s, p}+1\right)^{2}+(a-\lfloor a / U\rfloor) / U^{2}}}\right)
$$

where $U=M \omega_{s} T_{s}$.

\section{Secondary Users' Average Throughput Analysis}

4.1. SUs' Achievable Data Rate. During the off state, the maximum achievable data rate $\left(D_{s, 0}\right)$ for SUs under the effect of background noise and residual SI is

$$
D_{s, 0}=\log _{2}\left(1+\frac{\mathrm{SNR}_{s, s}}{1+(1-\kappa) \mathrm{SNR}_{s, s}}\right) .
$$

If the coordinator falsely detects that there is no primary activity in the on state, the achievable data rate $\left(D_{s, 1}\right)$ for SUs is

$$
D_{s, 1}=\log _{2}\left(1+\frac{\mathrm{SNR}_{s, s}}{1+\mathrm{SNR}_{s, p}+(1-\kappa) \mathrm{SNR}_{s, s}}\right) .
$$

In this paper, the effect of multiuser interference is assumed to be controlled and cancelled effectively, using physical layer and MAC layer techniques, for example, through adaptive beamforming as in [24], adaptive rate/power control and scheduling mechanisms as in $[25,26]$.

4.2. SUs' Average Throughput in Half-Duplex Mode. In order to compare our proposed full-duplex based sensing protocols to the existing protocols, in this subsection, we first introduce the SUs' average throughput in conventional half-duplex mode. As illustrated in Figure 3(a), there are four different states that should be considered to formulate the half-duplex (HD) SUs' average throughput $\left(\tau_{s, \mathrm{HD}}\right)$. As derived in [5], assuming asynchronicity between SUs and PUs, $\tau_{s, \mathrm{HD}}$ can be expressed as

$$
\tau_{s, \mathrm{HD}}=\sum_{i=00}^{11} P\left[S_{i, \mathrm{HD}}\right] C_{i, \mathrm{HD}}
$$

where $P\left[S_{i, \mathrm{HD}}\right], \forall i$ are defined as probability of event $S_{i, \mathrm{HD}}$ occurred in HD scheme, and following the assumed ON/OFF distributions, they can be expressed as

$$
\begin{aligned}
& P\left[S_{00, \mathrm{HD}}\right]=\frac{\rho_{\text {off }}}{\rho_{\text {off }}+\rho_{\text {on }}} e^{-T_{\mathrm{p}} / \rho_{\text {off }},} \\
& P\left[S_{01, \mathrm{HD}}\right]=\frac{\rho_{\text {off }}}{\rho_{\text {off }}+\rho_{\text {on }}}\left(1-e^{-T_{p} / \rho_{\text {off }}}\right), \\
& P\left[S_{10, \mathrm{HD}}\right]=\frac{\rho_{\text {on }}}{\rho_{\text {off }}+\rho_{\text {on }}}\left(1-e^{-T_{p} / \rho_{\text {on }}}\right), \\
& P\left[S_{11, \mathrm{HD}}\right]=\frac{\rho_{\text {on }}}{\rho_{\text {off }}+\rho_{\text {on }}} e^{-T_{p} / \rho_{\text {on }}} .
\end{aligned}
$$

In addition, $C_{i, \mathrm{HD}}$ is data rate for $S_{i, \mathrm{HD}}$ event for HD scheme, which can be found in [5].

4.3. SUs' Average Throughput in Full-Duplex Transmit-SenseReception Mode. Following the similar approach as in the HD scheme under the same four different cases, the average throughput for FDr scheme $\left(\tau_{s, \mathrm{FDr}}\right)$ can be obtained as

$$
\tau_{s, \mathrm{FDr}}=\sum_{i=00}^{11} P\left[S_{i, \mathrm{FDr}}\right] C_{i, \mathrm{FDr}}
$$

where $P\left[S_{i, \mathrm{FDr}}\right], \forall i$, are defined as probability of event $S_{i, \mathrm{FDr}}$ occurring in $\mathrm{FDr}$ scheme, and $C_{i, \mathrm{FDr}}$ is the achievable throughput for $S_{i, \mathrm{FDr}}$ event for FDr scheme. In this case, as illustrated in Figure 3(b), we have

$$
P\left[S_{i, \mathrm{FDr}}\right]=P\left[S_{i, \mathrm{HD}}\right], \quad \forall i .
$$

For an ideal single channel point-to-point communication, the achievable throughput in FDr is twice as high as that in HD scheme. However, due to the SI effect (when $0 \leq \kappa<1$ ) on $D_{s, 0}, D_{s, 1}$, and $P_{d}$, the achievable throughput will be lower 


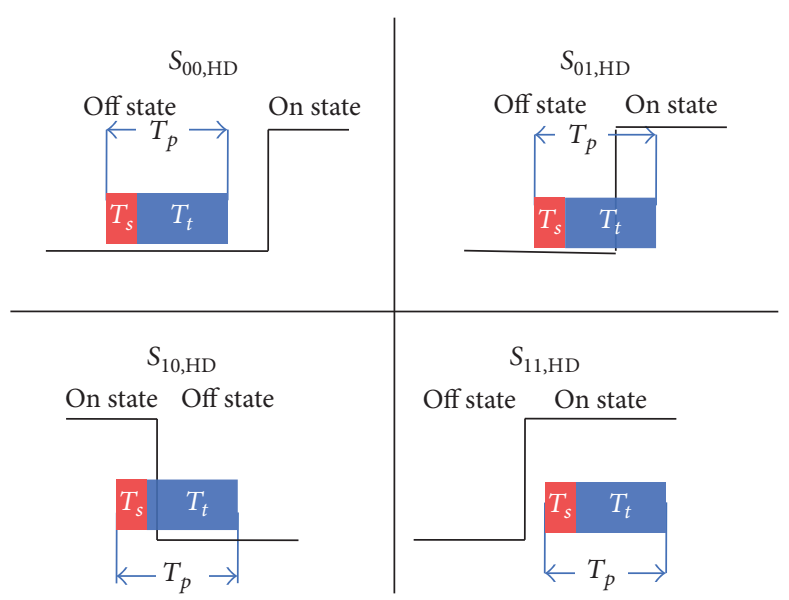

(a)

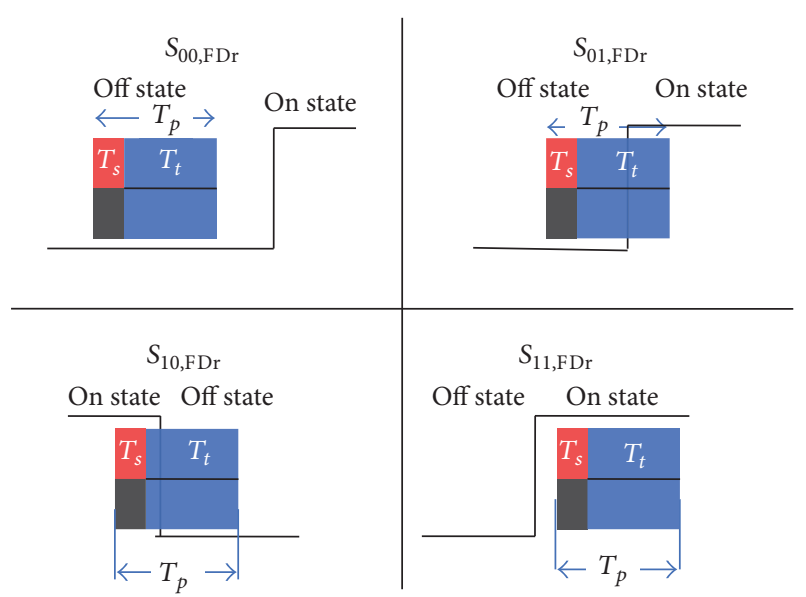

(b)

FIgURE 3: Four possible states in (a) HD and (b) FDr scenario.

than in the perfect SI cancellation case $(\kappa=1) . C_{i, \mathrm{FDr}}$ can be calculated as

$$
\begin{aligned}
& C_{00, \mathrm{FDr}}=2 \overline{P_{f}} \frac{T_{p}-T_{s}}{T_{p}} D_{s, 0} \\
& C_{01, \mathrm{FDr}} \\
& =\frac{2}{T_{p}} \frac{\left(D_{s, 0} \overline{P_{f}}-D_{s, 1} \overline{P_{d}}\right)\left(\rho_{\text {off }}-\left(T_{p}+\rho_{\text {off }}\right) e^{-T_{p} / \rho_{\text {off }}}\right)}{1-e^{-T_{p} / \rho_{\text {off }}}} \\
& \quad+\frac{2}{T_{p}}\left(\overline{P_{d}} D_{s, 1} T_{p}-\overline{P_{f}} D_{s, 0} T_{s}\right)
\end{aligned}
$$

$C_{10, \mathrm{FDr}}$

$$
\begin{aligned}
= & \frac{2}{T_{p}} \frac{\left(D_{s, 1} \overline{P_{d}}-D_{s, 0} \overline{P_{f}}\right)\left(\rho_{\mathrm{on}}-\left(T_{p}+\rho_{\mathrm{on}}\right) e^{-T_{p} / \rho_{\mathrm{on}}}\right)}{1-e^{-T_{p} / \rho_{\mathrm{on}}}} \\
& +\frac{2}{T_{p}}\left(\overline{P_{f}} D_{s, 0} T_{p}-\overline{P_{d}} D_{s, 1} T_{s}\right)
\end{aligned}
$$$$
C_{11, \mathrm{FDr}}=2 \overline{P_{d}} \frac{T_{p}-T_{s}}{T_{p}} D_{s, 1}
$$

where $\overline{P_{d}}=1-P_{d}$ and $\overline{P_{f}}=1-P_{f}$.

4.4. SUs' Average Throughput in Full-Duplex Transmit-Sense Mode. In contrast with $\mathrm{HD}$ and FDr schemes, in FDs, the transmission time during a frame is not constant. SUs continuously sense the channel and can immediately start or stop transmission based on the sensing result. Hence, only two states are studied for average throughput calculation as shown in Figure 4 . The probabilities of the events $S_{00, F D s}$ and
$S_{11, \mathrm{FDs}}$ are defined as the probability of being in off and on state, respectively, and they can be expressed as

$$
\begin{aligned}
& P\left[S_{00, \mathrm{FDs}}\right]=\frac{\rho_{\mathrm{off}}}{\rho_{\mathrm{off}}+\rho_{\mathrm{on}}} \\
& P\left[S_{11, \mathrm{FDs}}\right]=\frac{\rho_{\mathrm{on}}}{\rho_{\text {off }}+\rho_{\mathrm{on}}} .
\end{aligned}
$$
lated by

Average data rate during $S_{00 \text {,FDs }}$ and $S_{11, \mathrm{FDs}}$ can be calcu-

$$
\begin{aligned}
& C_{00, \mathrm{FDs}}=\overline{P_{f}} D_{s, 0} \\
& C_{11, \mathrm{FDs}}=\overline{P_{d}} D_{s, 1} .
\end{aligned}
$$

Therefore, SUs' average throughput $\left(\tau_{s, \mathrm{FDs}}\right)$ for FDs scheme is

$$
\tau_{s, \mathrm{FDs}}=\sum_{i=00}^{11} P\left[S_{i, \mathrm{FDs}}\right] C_{i, \mathrm{FDs}}
$$

\section{Primary Users' Average Throughput Analysis}

5.1. PUs' Achievable Data Rate. The PUs' achievable data rate without interference $\left(D_{p, 0}\right)$ from SU is

$$
D_{p, 0}=\log _{2}\left(1+\operatorname{SNR}_{p, p}\right) .
$$

The PUs' achievable data rate with interference $\left(D_{p, 1}\right)$ from SU due to miss-detection can be calculated as

$$
D_{p, 1}=\log _{2}\left(1+\frac{\mathrm{SNR}_{p, p}}{1+\mathrm{SNR}_{p, s}}\right)
$$

5.2. PUs' Average Throughput When Secondary Is in HalfDuplex or Full-Duplex Transmit-Sense-Reception Modes. The PUs' average throughput can be calculated by modeling the 


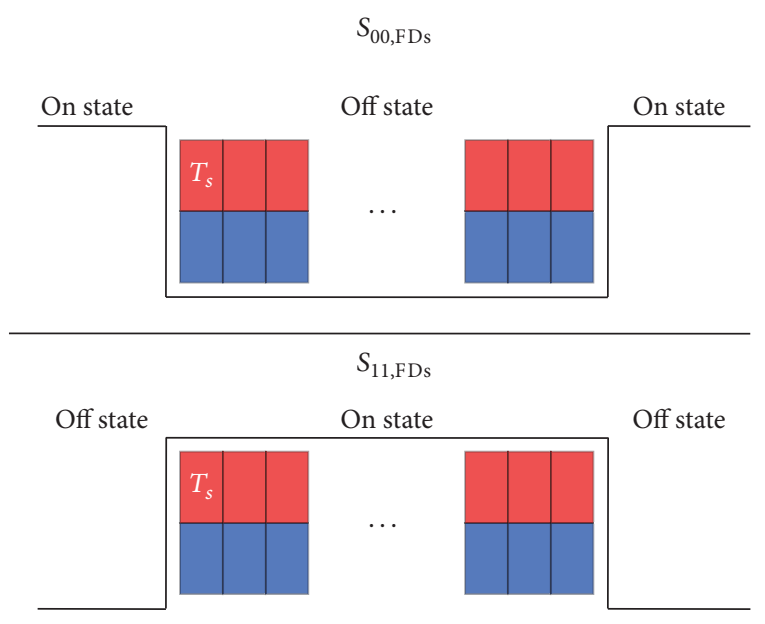

Figure 4: Two possible conditions in FDs scenario.

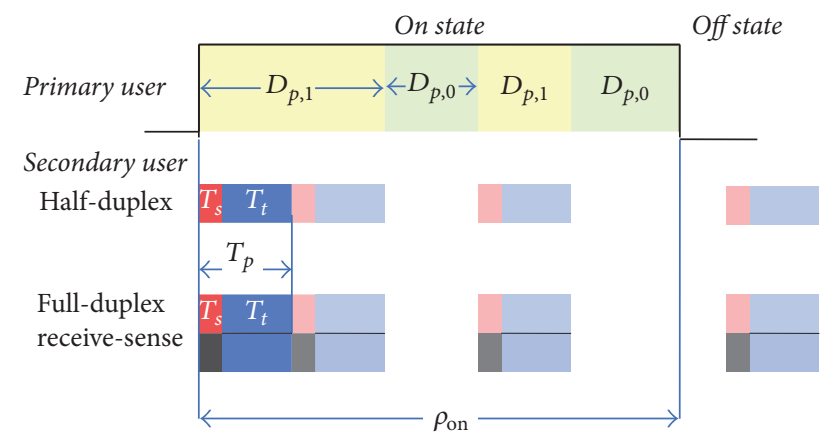

FIGURE 5: Illustration of PU's average throughput when SU uses HD or FDr schemes.

on state event as a time-slotted frame of size $T_{p}$, as shown in Figure 5. The average number of slots $\left(b_{T p}\right)$ is defined as ratio of the primary user on sate $\left(\rho_{\text {on }}\right)$ to sensing period $\left(T_{p}\right)$. Furthermore, the event where SUs transmit during the on state can be modeled as binomial distribution with the probability of occurrence given by $1-P_{d} \cdot P\left[S_{j, p}\right]$ is defined as probability that $j$ frames of SUs are transmitted during $\rho_{\text {on }}$ of PU when SUs use HD or FDr scheme, which can be expressed as

$$
P\left[S_{j, p}\right]=\left(\begin{array}{c}
b_{T_{p}} \\
j
\end{array}\right) \bar{P}_{d}^{j} P_{d}^{\left(b_{T p}-j\right)}
$$

where

$$
b_{T p}=\left[\frac{\rho_{\mathrm{on}}}{T_{p}}\right] \text {, }
$$

and $[\cdot]$ is rounded to nearest integer number operator. The average data rate $\left(C_{j, p}\right)$ when $j$ frames of SUs are transmitted during $\rho_{\text {on }}$ of PU can be calculated as

$$
C_{j, p}=\left(j \cdot D_{p, 1} \cdot \frac{\rho_{\text {on }}}{\rho_{\text {off }}+\rho_{\text {on }}} \cdot \frac{T_{p}}{\rho_{\text {on }}}\right)
$$

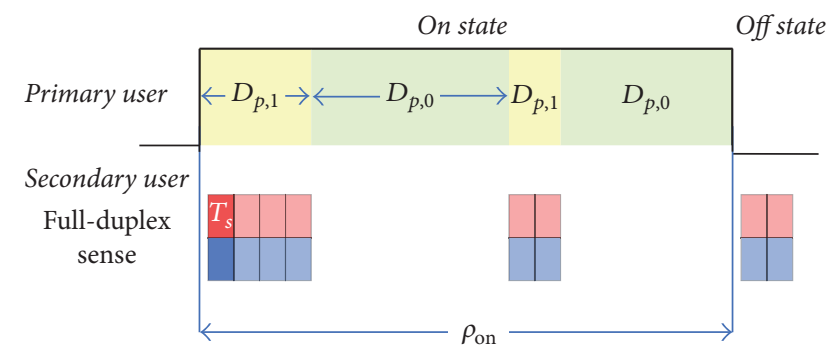

FIGURE 6: Illustration of PU's average throughput when SU uses FDs scheme.

$$
\begin{aligned}
& +\left(D_{p, 0} \cdot \frac{\rho_{\mathrm{on}}}{\rho_{\mathrm{off}}+\rho_{\mathrm{on}}} \cdot \frac{\rho_{\mathrm{on}}-j T_{p}}{\rho_{\mathrm{on}}}\right) \\
& =\frac{\rho_{\mathrm{on}} D_{p, 0}-j T_{p}\left(D_{p, 0}-D_{p, 1}\right)}{\rho_{\mathrm{off}}+\rho_{\mathrm{on}}} .
\end{aligned}
$$

Finally, the PUs' average throughput for $\mathrm{HD}\left(\tau_{p, \mathrm{HD}}\right)$ and $\operatorname{FDr}\left(\tau_{p, \mathrm{FDr}}\right)$ cases can be formulated as

$$
\tau_{p, \mathrm{HD}}=\tau_{p, \mathrm{FDr}}=\sum_{j=0}^{b_{T p}} P\left[S_{j, p}\right] C_{j, p} .
$$

5.3. PU's Average Throughput When Secondary User Is in Full-Duplex Transmit-Sense Mode. In the same fashion as in Section 5.2, PUs' average throughput when SUs use FDs scheme can be calculated as shown in Figure 6. Instead of dividing by $T_{p}, \rho_{\mathrm{on}}$ is divided by $T_{s}$ to estimate the number of slots, $b_{T s} . P\left[S_{l, p \mathrm{FDs}}\right]$ is defined as probability that $l$ frames of SUs are transmitted during $\rho_{\text {on }}$ of PU when SUs use FDs scheme, which can be expressed as

$$
P\left[S_{l, p \mathrm{FDs}}\right]=\left(\begin{array}{c}
b_{T_{s}} \\
l
\end{array}\right){\overrightarrow{P_{d}}}^{l} P_{d}^{\left(b_{T s}-l\right)},
$$

where

$$
b_{T s}=\left[\frac{\rho_{\mathrm{on}}}{T_{s}}\right] .
$$

The average data rate $\left(C_{l, p F D s}\right)$ when $l$ frames of SU are transmitted during $\rho_{\text {on }}$ of PU can be calculated as

$$
\begin{aligned}
C_{l, p \mathrm{FDs}}= & \left(l \cdot D_{p, 1} \cdot \frac{\rho_{\mathrm{on}}}{\rho_{\mathrm{off}}+\rho_{\mathrm{on}}} \cdot \frac{T_{s}}{\rho_{\mathrm{on}}}\right) \\
& +\left(D_{p, 0} \cdot \frac{\rho_{\mathrm{on}}}{\rho_{\mathrm{off}}+\rho_{\mathrm{on}}} \cdot \frac{\rho_{\mathrm{on}}-l T_{s}}{\rho_{\mathrm{on}}}\right) \\
= & \frac{\rho_{\mathrm{on}} D_{p, 0}-l T_{s}\left(D_{p, 0}-D_{p, 1}\right)}{\rho_{\mathrm{off}}+\rho_{\mathrm{on}}} .
\end{aligned}
$$

The PU's average throughput when SUs use FDs scheme $\left(\tau_{p, \text { FDs }}\right)$ can be calculated as

$$
\tau_{p, \mathrm{FDs}}=\sum_{l=0}^{b_{T s}} P\left[S_{l, p \mathrm{FDs}}\right] C_{l, \mathrm{pFDs}} .
$$


TABLE 1: Proposed MAC simulation parameters.

\begin{tabular}{lcl}
\hline Parameter & Value & Description \\
\hline$T_{p}$ & $32 \mathrm{~ms}$ & Sensing period \\
$\kappa$ & 0.99 & Self-interference mitigation coefficient \\
$W$ & $11[28]$ & Number of primary user's channels \\
$\varepsilon_{\mathrm{th}} / \sigma^{2}$ & $1.03[5]$ & Received signal power-to-noise ratio threshold \\
$\mathrm{SNR}_{s, s}$ & $10 \mathrm{~dB}[5]$ & Average signal-to-noise ratio received by secondary user from secondary signal \\
$\mathrm{SNR}_{s, p}$ & $-10 \mathrm{~dB}[5]$ & Average signal-to-noise ratio received by secondary user from primary signal \\
$\mathrm{SNR}_{p, p}$ & $10 \mathrm{~dB}$ & Average signal-to-noise ratio received by primary user from primary signal \\
$\mathrm{SNR}_{p, s}$ & $-10 \mathrm{~dB}$ & Average signal-to-noise ratio received by primary user from secondary signal \\
$\omega_{s}$ & $100 \mathrm{kHz}$ & Sampling rate \\
$\rho_{\text {off }}$ & {$[5]$} & Average length of off state for primary user \\
$\rho_{\mathrm{on}}$ & $640 \mathrm{~ms}$ & Average length of on state for primary user \\
\hline
\end{tabular}

\section{Analysis for Physical Layer Method and Numerical Results}

Based on the above derived average throughputs for both secondary and primary users per channel, the generalised average throughput for multichannel case can be formulated by inserting (14), (19), and (25) into (5), respectively, for secondary users. As discussed in Section 2, for the primary users, the average throughput for multichannel case is equal to the ones for single channel case, which are given by (31) and (35). Then, the optimization problems which aim to maximize the secondary users average throughput can be expressed as

$$
\begin{array}{ll}
\max _{m, v, T_{s}} & \tau_{s, \text { scheme }}^{(v)}=\frac{L \cdot \tau_{s, \text { scheme }}}{W}, \\
\text { s.t. } & \tau_{p, \text { scheme }}^{(v)} \geq \bar{R}_{p, \text { scheme }} \\
& \operatorname{SNR}_{x, y} \leq \overline{\operatorname{SNR}}_{x, y}, \quad \forall x, y,
\end{array}
$$

where $m(\leq M)$ is number of active secondary users, $\bar{R}_{p \text {,scheme }}$ is primary user's minimum rate constraint, and $\overline{\mathrm{SNR}}_{x, y}$ is the SNR upper limit for $(x, y)$ link. In order to obtain the optimal solution of problem (36), we can implement the first-order derivation of the objective function with respect to either $m, v$, or $T_{s}$ and then set the derived equation to zero if there is unique root. Alternatively, numerical results can be implemented to help to find the optimal solution. In the following subsections, numerical results are provided for both primary and secondary users, and the parameters used in the numerical results are shown in Table 1, which are in line with those in [5] for fair comparison.

6.1. Secondary Users' Average Throughput. In Figure 7, SUs' average throughput is presented versus number of cooperating SUs $(M)$ for different schemes and $\kappa$ values. It shows that FDr scheme achieves higher average throughput for SUs compared to FDs and HD. This is due to the longer transmitting time $\left(T_{t}\right)$ in FDr compared to the other schemes.

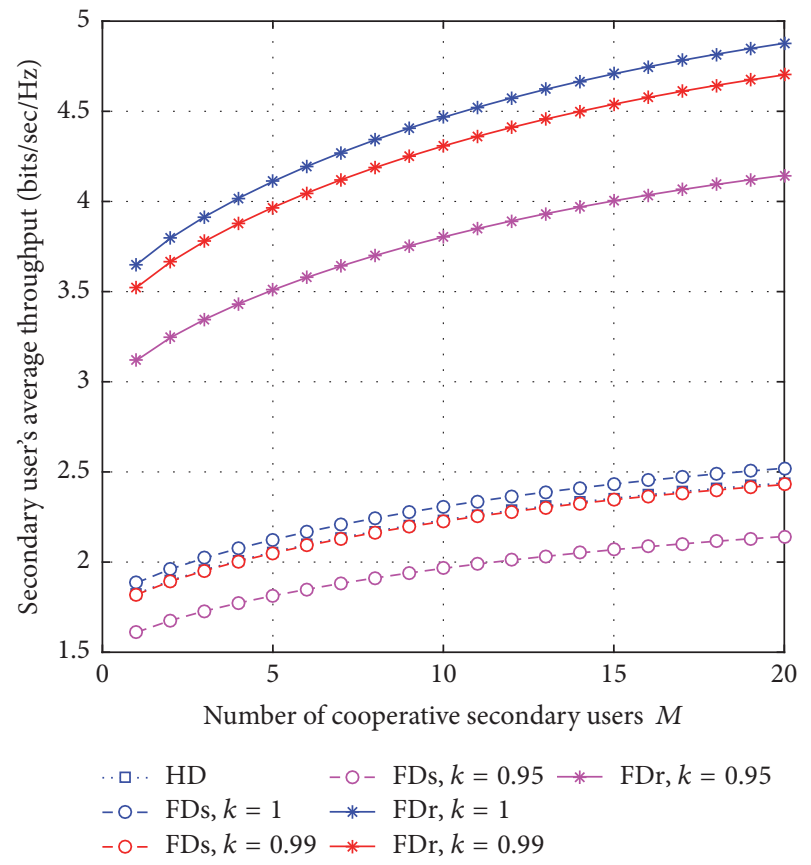

FIGURE 7: SU's average throughput versus $M$ for different schemes and different value of $\kappa$.

The SU's average throughput of different schemes monotonically increases with the number of cooperating SUs, $M$. As expected, it shows that cooperative sensing offers better performance compared to the noncooperative case $(M=$ $1)$. This figure also demonstrates the effect of $\kappa$ on the SUs' average throughput for FDr and FDs schemes, noting that in HD scheme SI is zero $(\kappa=1)$. By decreasing $\kappa$, the average throughput for both FDs and FDr deteriorates slightly.

6.2. Primary Users' Average Throughput. Figure 8 shows the average throughput of PU versus the number of SUs $(M)$ for different schemes and $\kappa$ values. Although for the SUs' average throughput, FDr outperforms FDs and HD, for the PUs' average throughput FDr gives the worse performance, especially 


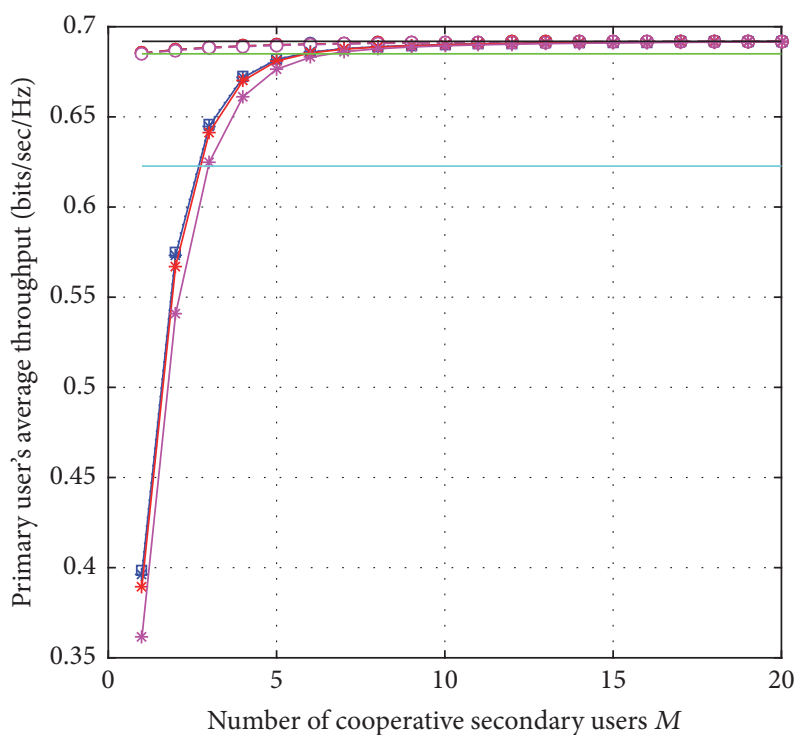

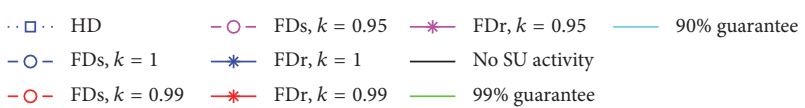

FIGURE 8: PU's average throughput versus $M$ for different schemes and $\kappa$.

for lower $M$. Indeed, it shows an average throughput trade-off between SUs and PUs. FDs gives similar average throughput performance as no SUs active case even the number of cooperative SUs is equal to one. This is because secondary users incorporate continuous sensing, and cooperation in this case does not provide much performance improvement for primary users. Furthermore, it can reduce transmitting time of the SU, $T_{t}$ during on state. As a result, PUs can transmit in the absence of SUs for most of the time during the on state.

According to this figure, the average throughput of $\mathrm{PU}$ increases with $M$ especially when SUs employ FDr or HD schemes. It shows that the use of cooperative sensing outperforms noncooperative sensing from both PU and SU points of view. The graphs also reveal that $\kappa$ plays an important role in PU's performance. In FDr case, a significant gain in PU's average throughput is achieved for higher values of $\kappa$. The reason is that the higher $\kappa$ would increase the $P_{d}$, which is in turn closely related to the average throughput of the PUs. When $P_{d}$ increases, the average throughput of PUs will increase. As seen from the figure, the PUs' average throughput for $\mathrm{HD}$ case is the same as for FDr when $\kappa$ is equal to one.

6.3. Multi-Channel Sensing Results. In Figure 9, SUs' average throughput in multichannel sensing case is presented versus number of cooperating SUs $(M)$ for different schemes, with $\kappa=0.99, W=11, T_{\text {so }}=1 \mathrm{~ms}$, and $\beta=0.2$. What is shown here is that an increasing number of channels, $V$, sensed by SUs will increase the average throughput. This is due to the fact that increasing $V$ will increase the sensing time $T_{s}$, so that the probability that SUs detect idle channels is increased as well. In addition, according to this figure, FDr

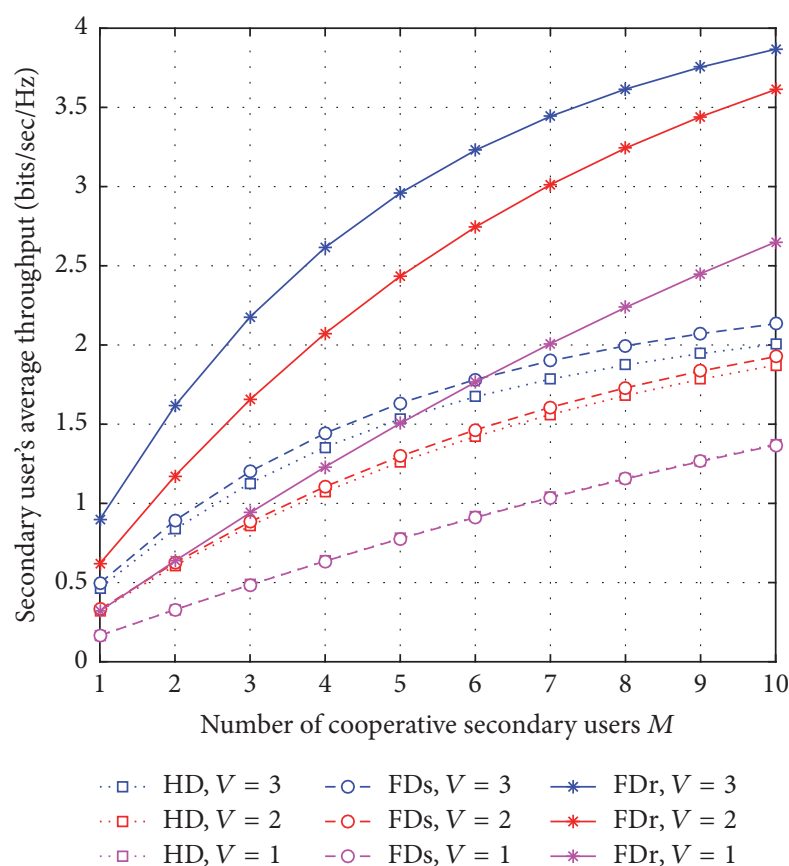

FIGURE 9: SU's average throughput versus $M$ for different schemes and values for $V$ and with $\kappa=0.99, W=11, T_{\text {so }}=1 \mathrm{~ms}$, and $\beta=0.2$.

still outperforms HD and FDs schemes for the multichannel sensing case. When $M$ increases, average throughput will also increase. By increasing $M$, the false alarm probability will be reduced, while the number of sensed idle channels will increase. This is consistent with our previous results (Figure 7). It is worthwhile to note that, for multichannel sensing case, the sensing time $T_{s}$ increases as the number of multichannels increase. In this case, with a fixed $T_{p}$, the detection probability $P_{d}$ will increase and $b_{T_{s}}$ in (33) will decrease, so that the primary users' average throughput will be affected.

\section{Proposed MAC Protocol Design}

7.1. Deployment Architecture. Our MAC design is based on a limited infrastructure support architecture in cognitive vehicular networks [27]. Road side units (RSU), as defined in IEEE 802.11p standard, are placed on the road. These play the role of coordinator nodes in the cooperative cognitive radio network, taking care of spectrum selection and access. On the other hand, vehicular nodes act as secondary users (SUs). Figure 10 illustrates the network architecture for our proposed MAC.

7.2. Proposed MAC Framework. Our MAC framework is developed based on a slotted time MAC structure illustrated in Figure 11. There is one control channel (CCC) and $W$ primary licensed channels, within $T_{p}$ time duration. Moreover, the proposed MAC protocol is divided into four phases.

The first phase is sensing phase (SP). Each SU which has packets to send senses $V$ channels from $W$ licensed channels during this phase. Energy detection technique is 


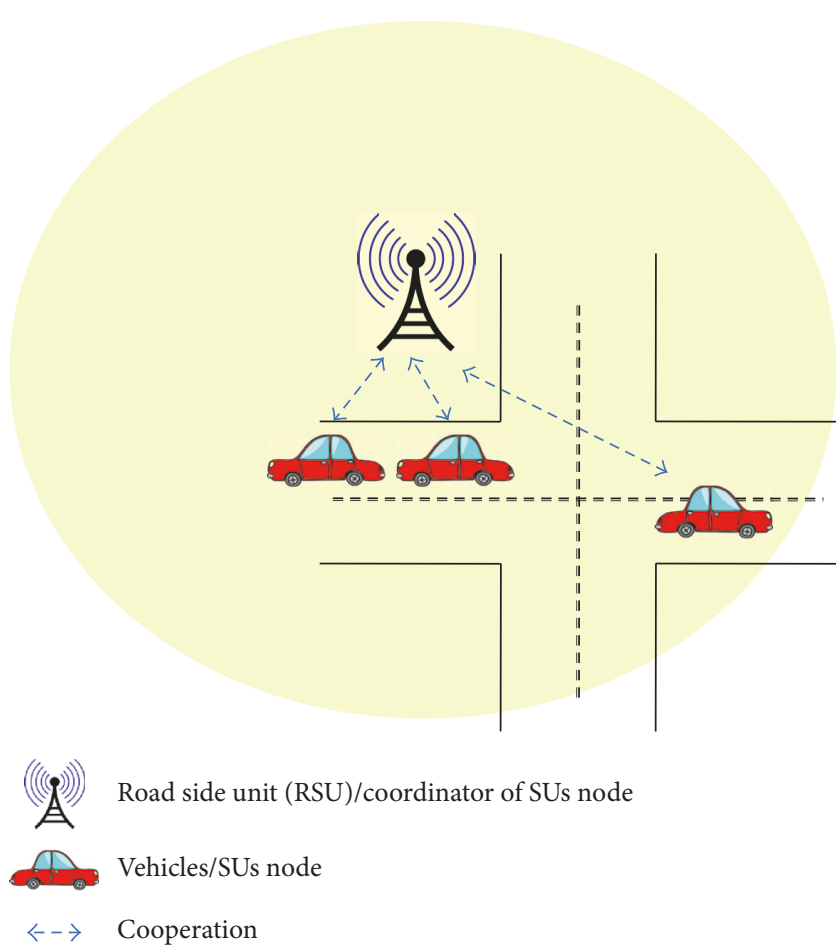

FIGURE 10: MAC deployment topology.

used to detect PU's activity. Furthermore, SUs listen to CCC for broadcast information.

The second phase is reporting and contending phase (RCP). In this phase, the SU informs the coordinator about the sensing result and its intention to use licensed channels. The CCC frame is split into $M_{x}$ minislots. Each SU selects one minislot based on the broadcast information received during the SP phase.

The third phase is the broadcast phase (BP). After receiving the sensing result information, the coordinator performs spectrum decision, access and SU management. Spectrum decision is performed by selecting idle channels based on overall energy statistic calculation as in [5]. Furthermore, $L$ identified available licensed channels are allocated among $M_{c}$ SUs. If $L<M_{c}$, only the first of $L$ SUs can use one channel per user. The remaining SUs will be allocated in the next time slot. If $L>M_{c}$, then each SU may be eligible for $\left\lfloor L / M_{c}\right\rfloor$ channels. $\lfloor\cdot\rfloor$ is rounded down to nearest integer operator. In relation to management of SUs, the coordinator removes inactive SUs and adds new SUs into its list of $M_{c}$ SUs. In addition, broadcast is also used for acknowledging arrival of new SUs.

Broadcast messages contain the following information:

(1) Number of current SUs in the cooperative network $\left(M_{c}\right)$ : this information is required for the new SU to join the cooperative network. The new SU selects a random minislot number from $M_{c}+1$ to $M_{x}$.

(2) Available licensed channels for specific SUs: transmission mode, FDr or FDs, according to Figure 11 is decided by the coordinator based on $M_{c}$ values. $M_{\text {th }}$ is defined as a threshold which allows each SU to use FDr mode based on PU's performance guarantee. Coordinator selects FDs transmission mode by default. However, If $M_{c}>M_{\text {th }}$ and both the coordinator and the SU have packets to send, then FDr can be selected.

(3) Synchronization information for all SUs in the cooperative network.

The last phase is data transmission phase (DTP). If an SU uses FDr mode, then data and acknowledgement can be transmitted in both uplink and downlink directions. FDs mode allows SU to send data in uplink or downlink direction and sense at the same time. During the DTP phase, if the SU detects primary user activity, it will stop transmitting the current data or acknowledgement.

7.3. Proposed MAC Protocol Average Throughput. In this section, the proposed MAC is evaluated using average throughput as performance metric.

7.3.1. Proposed MAC's Average Throughput Using Full-Duplex Transmit-Sense-Reception Mode. Average throughput can be calculated using the same method as in Section 4.3. However, average throughput calculation in the proposed MAC requires preparation time $\left(T_{\mathrm{pr}}\right)$, which consists of sensing time $\left(T_{s}\right)$, reporting time $\left(T_{r}\right)$, and broadcasting time $\left(T_{b}\right)$. Figure 12 shows the frame structure of the proposed MAC.

$$
T_{\mathrm{pr}, \mathrm{FDr}}=T_{s}+T_{r}+T_{b}=V \cdot T_{\mathrm{so}}+M_{x} \cdot T_{\mathrm{ro}}+T_{b} .
$$

The average throughput for the proposed MAC $\left(\tau_{s, \text { MFDr }}\right)$ can be calculated as

$$
\tau_{s, \mathrm{MFDr}}^{\left(V, M_{x}\right)}=\frac{L \cdot \sum_{i=00}^{11} P\left[S_{i, \mathrm{FDr}}\right] C_{i, \mathrm{MFDr}}}{W},
$$

where

$$
\begin{aligned}
& C_{00, \mathrm{MFDr}}=2 \overline{P_{f}} \frac{T_{p}-T_{\mathrm{pr}, \mathrm{FDr}}}{T_{p}} D_{s, 0} \\
& C_{01, \mathrm{MFDr}} \\
& =\frac{2}{T_{p}\left(1-e^{\left.-T_{p} / \rho_{\text {off }}\right)} D_{s, 0} \overline{P_{f}}\right.} \\
& \quad-\frac{2 D_{s, 1} \overline{P_{d}}\left(\rho_{\text {off }}-\left(T_{p}+\rho_{\text {off }}\right) e^{-T_{p} / \rho_{\text {off }}}\right)}{T_{p}\left(1-e^{-T_{p} / \rho_{\text {off }}}\right)}
\end{aligned}
$$




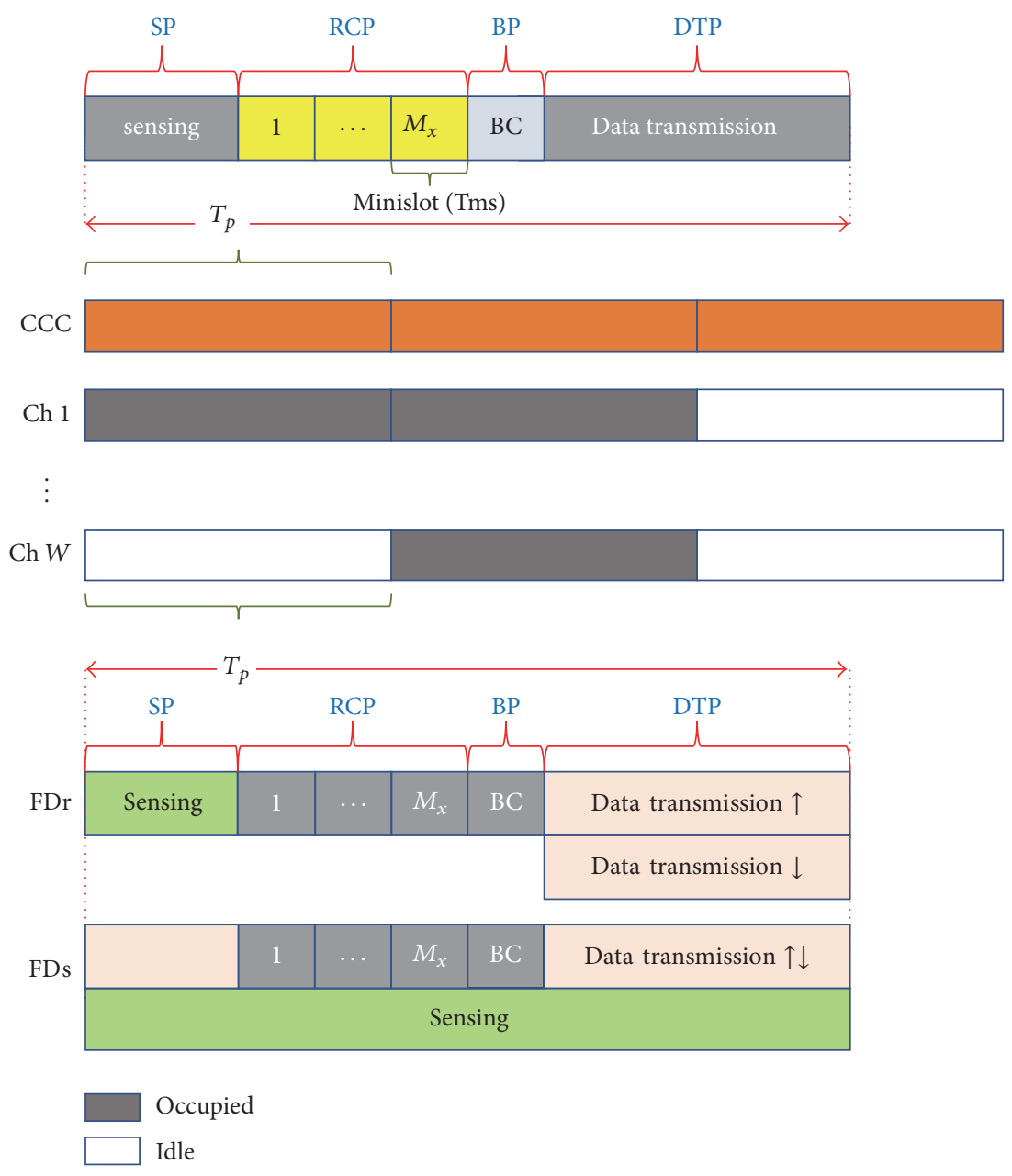

FIGURE 11: Proposed MAC framework.

$$
+\frac{2}{T_{p}}\left(\overline{P_{d}} D_{s, 1} T_{p}-\overline{P_{f}} D_{s, 0} T_{\mathrm{pr}, \mathrm{FDr}}\right)
$$

$C_{10, \mathrm{MFDr}}$

$$
\begin{aligned}
= & \frac{2}{T_{p}} \frac{D_{s, 1} \overline{P_{d}}-D_{s, 0} \overline{P_{f}}\left(\rho_{\mathrm{on}}-\left(T_{p}+\rho_{\mathrm{on}}\right) e^{-T_{p} / \rho_{\mathrm{on}}}\right)}{1-e^{-T_{p} / \rho_{\mathrm{on}}}} \\
& +\frac{2}{T_{p}}\left(\overline{P_{f}} D_{s, 0} T_{p}-\overline{P_{d}} D_{s, 1} T_{\mathrm{pr}, \mathrm{FDr}}\right) \\
C_{11, \mathrm{MFDr}} & =2 \overline{P_{d}} \frac{T_{p}-T_{\mathrm{pr}, \mathrm{FDr}}}{T_{p}} D_{s, 1} .
\end{aligned}
$$

$C_{i, \mathrm{MFDr}}$ is the achievable throughput of the proposed MAC for $S_{i, F D r}$ event in the FDr scheme.

7.3.2. Proposed MAC's Average Throughput Using the FullDuplex Transmit-Sense Mode. In FDs scheme, $T_{\mathrm{pr}}$ only consists of reporting $\left(T_{s}\right)$ and broadcasting time $\left(T_{b}\right)$, because sensing time $\left(T_{s}\right)$ is in parallel with transmission time $\left(T_{t}\right)$.

$$
T_{\mathrm{pr}, \mathrm{FDs}}=T_{r}+T_{b}=M_{x} \cdot T_{\mathrm{ro}}+T_{b} \text {. }
$$

Following the same method as in Section 4.4, the average throughput for the proposed MAC can be calculated as

$$
\tau_{s, \mathrm{MFDs}}^{\left(\mathrm{V}, M_{x}\right)}=\frac{L \cdot \sum_{i=00}^{11} P\left[S_{i, \mathrm{FDs}}\right] C_{i, \mathrm{MFDs}}}{W},
$$

where $C_{i, \mathrm{MFDs}}$ is the achievable throughput of our proposed MAC for $S_{i, \mathrm{FDs}}$ event for FDs scheme

$$
\begin{aligned}
C_{00, \mathrm{MFDs}}= & \frac{\overline{P_{f}} D_{s, 0}\left(T_{p}-T_{\mathrm{pr}, \mathrm{FDs}}\right)}{T_{p}}, \\
C_{11, \mathrm{MFDs}}= & \frac{\overline{P_{d}} D_{s, 1}\left(T_{p}-T_{\mathrm{pr}, \mathrm{FDs}}\right)}{T_{p}} .
\end{aligned}
$$

7.4. Proposed MAC Protocol Numerical Results and Analysis. Tables 1 and 2 summarize the parameters for evaluation of our proposed MAC protocol. In order to perform an evaluation based on the realistic scenario (i.e., utilizing TV white space channels), the number of primary channels $(W)$ is established based on system B TV channels in Western Europe and many other countries in Africa, Asia, and the Pacific [28]. It is 


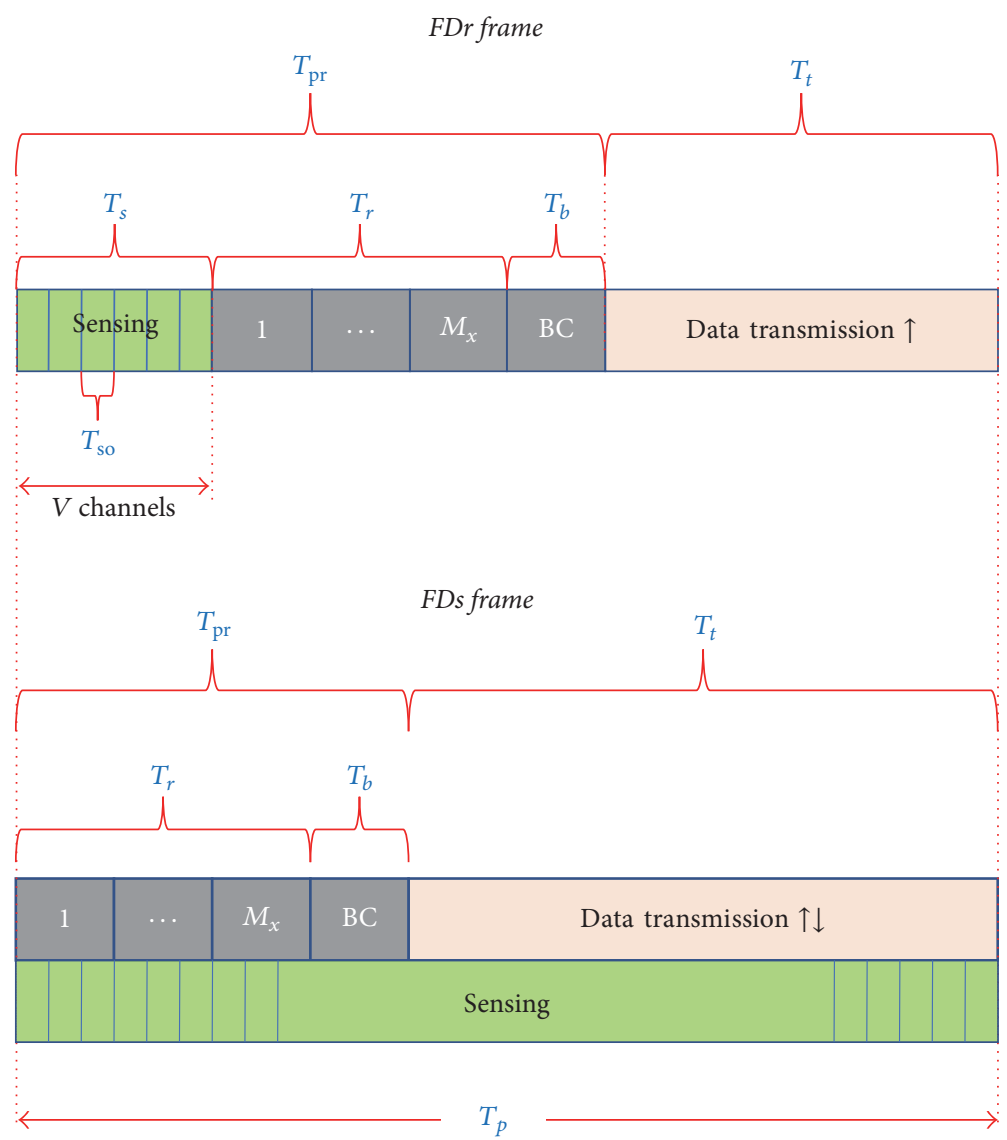

Figure 12: Proposed MAC frame structure.

TABLE 2: Proposed MAC simulation parameters.

\begin{tabular}{lcc}
\hline Parameter & Value & Description \\
\hline$T_{p}$ & $32 \mathrm{~ms}$ & Sensing period \\
$T_{\text {so }}$ & $1 \mathrm{~ms}$ & Sensing time of each channel \\
$T_{\text {ro }}$ & $0.5 \mathrm{~ms}$ & Reporting time for one minislot \\
$T_{b}$ & $2 \mathrm{~ms}$ & Broadcasting time \\
\hline
\end{tabular}

assumed the cooperative networks are saturated when the number of cooperative users $(M)$ is equal to the maximum number of minislot $\left(M_{x}\right)$.

Figure 13 demonstrates average throughput of the proposed MAC for various number of channels sensed by SU $(V)$ and different number of minislots $\left(M_{x}\right)$. It shows FDr scheme has a higher average throughput compared to FDs scheme. In both schemes, increasing $M_{x}$ improves average throughput until the optimum value of $M_{x}$. It deteriorates slightly after reaching the optimum value. Here, $M_{x}$ can be linked with the number of cooperative users which have been activated. Specifically, $M_{x}$ increases throughput by improving the number of SUs $(M)$ that perform cooperative spectrum sensing. Furthermore, cooperative spectrum sensing improves the average throughput. At the same time, minislots consume

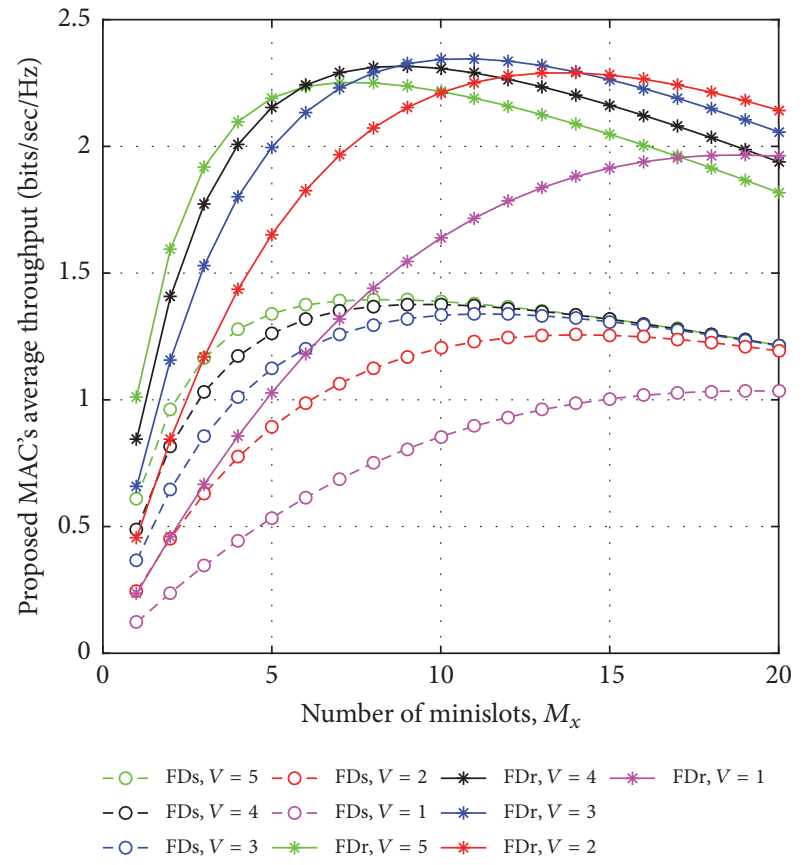

FIgURE 13: Proposed MAC's average throughput versus $M_{x}$ for different schemes and value for $V$. 
allocated time in a framework which cannot be used for data transmission. As a result, increasing $M_{x}$ shortens the transmission time in one frame. In general, when throughput gain from cooperative spectrum sensing cannot compensate for throughput loss due to allocated minislot in a frame, it reduces the average throughput.

The number of sensed channels $(V)$ has a different effect for FDr and FDs schemes. In FDs scheme, increasing the value of $V$ slightly improves the average throughput. This is due to the fact that increasing $V$ will increase the probability that SUs detect idle channels. Different from FDs scheme which only has throughput gain, in FDr scheme, there is throughput loss due to sensing time. Sensing time is considered as nontransmitting time, which reduces data transmission time. As a result, an increment of $V$ will decrease slightly the average throughput. When throughput gain cannot compensate for throughput loss, increasing $V$ deteriorates the average throughput.

Based on the numerical results, the optimum values for $V$ and $M_{x}$ are shown to be 3 and 11, respectively. It produces the maximum average throughput of $2.3436 \mathrm{bits} / \mathrm{sec} / \mathrm{Hz}$ when operating in FDr mode and $1.3387 \mathrm{bits} / \mathrm{sec} / \mathrm{Hz}$ in FDs mode. In other words, the stated parameter values can be implemented for optimum proposed MAC protocol in the cooperative cognitive network, while utilizing system B TV channels. It is worthwhile to note that our proposed cooperative full-duplex spectrum sensing technique needs to set up a coordinator to allocate the spectrum resource to the SUs. Such scheme is quite different with the distributed user contention based resource allocation (e.g., see [9]). In this case, fair comparison between these schemes is difficult to obtain. In addition, like the work in [9], the author proposed the frame fragmentation during the data transmission phase in order to protect the PUs. Such design makes the data transmission model quite different from ours. On the other hand, we compare our proposed full-duplex scenarios with the conventional half-duplex scheme in order to show the performance improvement.

\section{Conclusion}

Performance trade-offs for FDr, FDs, and HD schemes are found by analyzing the average throughput of both PUs and SUs under multichannel spectrum sharing and considering the effect of residual self-interference. The FDr scheme can offer similar achievable PU throughput as in FDs by incorporating a sufficient number of cooperating SUs for full-duplex cooperative sensing. In addition, it is shown that the result is consistent for different primary channel utilization and parameters setup. Furthermore, the proposed MAC protocol based on numerical results is designed and evaluated. The optimum parameter sets for proposed MAC are found to be implemented in particular cognitive networks in the future (e.g., the cognitive vehicular network by utilizing system B TV channels).

\section{Conflicts of Interest}

The authors declare that they have no conflicts of interest.

\section{Acknowledgments}

This work was partially supported by the Engineering and Physical Science Research Council (EPSRC) through the SENSE Grant EP/P003486/1.

\section{References}

[1] J. Mitola III and G. Q. Maguire Jr., "Cognitive radio: making software radios more personal," IEEE Personal Communications, vol. 6, no. 4, pp. 13-18, 1999.

[2] S. Haykin, "Cognitive radio: brain-empowered wireless communications," IEEE Journal on Selected Areas in Communications, vol. 23, no. 2, pp. 201-220, 2005.

[3] A. Shadmand, K. Nehra, and M. Shikh-Bahaei, "Cross-layer design in dynamic spectrum sharing systems," EURASIP Journal on Wireless Communications and Networking, vol. 2010, article 458472, 2010.

[4] C. Jiang, N. C. Beaulieu, L. Zhang, Y. Ren, M. Peng, and H.-H. Chen, "Cognitive radio networks with asynchronous spectrum sensing and access," IEEE Network, vol. 29, no. 3, pp. 88-95, 2015.

[5] C. Jiang, N. C. Beaulieu, and C. Jiang, "A novel asynchronous cooperative spectrum sensing scheme," in Proceedings of the 2013 IEEE International Conference on Communications, ICC 2013, pp. 2606-2611, Hungary, June 2013.

[6] C. Jiang, C. Jiang, and N. C. Beaulieu, "A contention-based wideband DSA algorithm with asynchronous cooperative spectrum sensing," in Proceedings of the 2013 IEEE Global Communications Conference, GLOBECOM 2013, pp. 1069-1074, USA, December 2013.

[7] A. Sabharwal, P. Schniter, D. Guo, D. W. Bliss, S. Rangarajan, and R. Wichman, "In-band full-duplex wireless: challenges and opportunities," IEEE Journal on Selected Areas in Communications, vol. 32, no. 9, pp. 1637-1652, 2014.

[8] W. Cheng, X. Zhang, and H. Zhang, "Full-duplex spectrumsensing and MAC-protocol for multichannel nontime-slotted cognitive radio networks," IEEE Journal on Selected Areas in Communications, vol. 33, no. 5, pp. 820-831, 2015.

[9] L. T. Tan and L. B. Le, "Distributed MAC Protocol Design for Full-Duplex Cognitive Radio Networks," in Proceedings of the GLOBECOM 2015 - 2015 IEEE Global Communications Conference, pp. 1-6, San Diego, CA, USA, December 2015.

[10] Y. Liao, T. Wang, L. Song, and Z. Han, "Listen-and-Talk: Protocol Design and Analysis for Full-Duplex Cognitive Radio Networks," IEEE Transactions on Vehicular Technology, vol. 66, no. 1, pp. 656-667, 2017.

[11] W. Afifi and M. Krunz, "Incorporating Self-Interference Suppression for Full-duplex Operation in Opportunistic Spectrum Access Systems," IEEE Transactions on Wireless Communications, vol. 14, no. 4, pp. 2180-2191, 2015.

[12] L. T. Tan and L. B. Le, "Design and optimal configuration of fullduplex MAC protocol for cognitive radio networks considering self-interference," IEEE Access, vol. 3, pp. 2715-2729, 2015.

[13] W. Afifi and M. Krunz, "Adaptive transmission-receptionsensing strategy for cognitive radios with full-duplex capabilities," in Proceedings of the 2014 IEEE International Symposium on Dynamic Spectrum Access Networks, DYSPAN 2014, pp. 149160, USA, April 2014.

[14] Y. Liao, T. Wang, L. Song, and B. Jiao, "Cooperative spectrum sensing for full-duplex cognitive radio networks," in Proceedings 
of the 2014 IEEE International Conference on Communication Systems, IEEE ICCS 2014, pp. 56-60, China, November 2014.

[15] V. Towhidlou and M. Shikh-Bahaei, "Cooperative ARQ in full duplex cognitive radio networks," in Proceedings of the 27th IEEE Annual International Symposium on Personal, Indoor, and Mobile Radio Communications, PIMRC 2016, Spain, September 2016.

[16] S. Ha, W. Lee, J. Kang, and J. Kang, "Cooperative spectrum sensing in non-time-slotted full duplex cognitive radio networks," in Proceedings of the 13th IEEE Annual Consumer Communications and Networking Conference, CCNC 2016, pp. 820-823, usa, January 2016.

[17] T. Febrianto and M. Shikh-Bahaei, "Optimal full-duplex cooperative spectrum sensing in asynchronous cognitive networks," in Proceedings of the 3rd IEEE Asia Pacific Conference on Wireless and Mobile, APWiMob 2016, pp. 1-6, Indonesia, September 2016.

[18] L. T. Tan and L. B. Le, "Multi-channel MAC protocol for fullduplex cognitive radio networks with optimized access control and load balancing," in Proceedings of the 2016 IEEE International Conference on Communications, ICC 2016, Malaysia, May 2016.

[19] T.-N. Hoan, V.-V. Hiep, and I.-S. Koo, "Multichannel-sensing scheduling and transmission-energy optimizing in cognitive radio networks with energy harvesting," Sensors, vol. 16, no. 4, article no. 461, 2016.

[20] G. Bianchi, "Performance analysis of the IEEE 802.11 distributed coordination function," IEEE Journal on Selected Areas in Communications, vol. 18, no. 3, pp. 535-547, 2000.

[21] C. Jiang, Y. Chen, and K. J. R. Liu, "A renewal-theoretical framework for dynamic spectrum access with unknown primary behavior," in Proceedings of the 2012 IEEE Global Communications Conference, GLOBECOM 2012, pp. 1422-1427, USA, December 2012.

[22] K. J. R. Liu and B. Wang, "Cognitive radio networking and security: A Game-Theoretic view," Cognitive Radio Networking and Security: A Game-Theoretic View, pp. 1-601, 2010.

[23] W. Cheng, X. Zhang, and H. Zhang, "Optimal dynamic power control for full-duplex bidirectional-channel based wireless networks," in Proceedings of the 32nd IEEE Conference on Computer Communications, IEEE INFOCOM 2013, pp. 31203128, Italy, April 2013.

[24] J. Hou, N. Yi, and Y. Ma, "Joint space-frequency user scheduling for MIMO random beamforming with limited feedback," IEEE Transactions on Communications, vol. 63, no. 6, pp. 2224-2236, 2015.

[25] A. Shadmand and M. Shikh-Bahaei, "Multi-user timefrequency downlink scheduling and resource allocation for LTE cellular systems," in Proceedings of the IEEE Wireless Communications and Networking Conference 2010, WCNC 2010, Australia, April 2010.

[26] A. Kobravi and M. Shikh-Bahaei, "Cross-layer adaptive ARQ and modulation tradeoffs," in Proceedings of the 18th Annual IEEE International Symposium on Personal, Indoor and Mobile Radio Communications, PIMRC'07, Greece, September 2007.

[27] S. Basagni, M. Conti, S. Giordano, and I. Stojmenovic, "Mobile Ad Hoc Networking: Cutting Edge Directions: Second Edition," Mobile Ad Hoc Networking: Cutting Edge Directions: Second Edition, 2013.

[28] IEEE Standard for Information Technology-Telecommunications and Information Exchange between System Wireless
Regional Area Networks (WRAN)-Specific Requirements-Part 22: Cognitive Wireless RAN Medium Access Control (MAC) and Physical Layer (PHY) Specifications: Policies and Procedures for Operation in the TV bands, IEEE Standard 802.22 https://standards.ieee.org/about/get/802/802.22.html, 2011. 


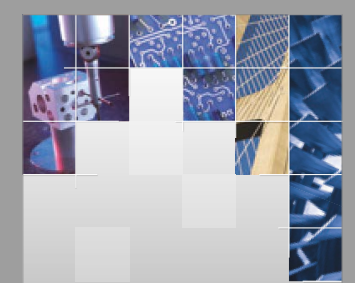

\section{Enfincering}
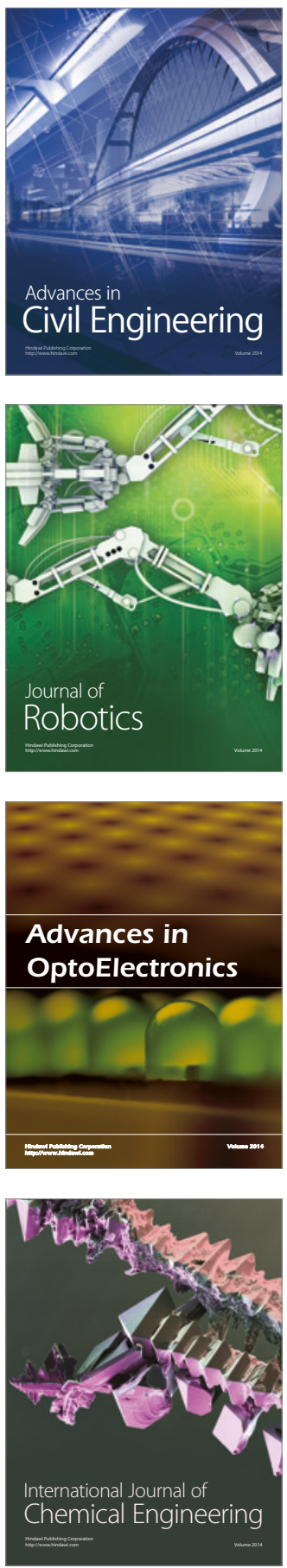

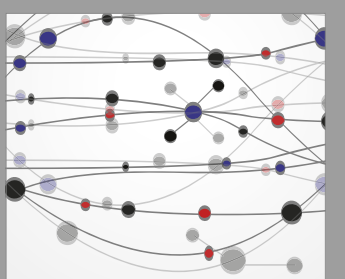

The Scientific World Journal

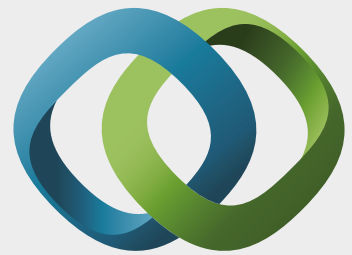

\section{Hindawi}

Submit your manuscripts at

https://www.hindawi.com
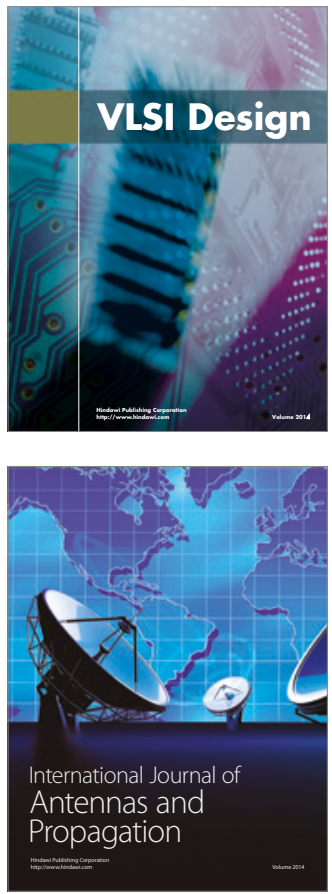

\section{Rotating}

Machinery
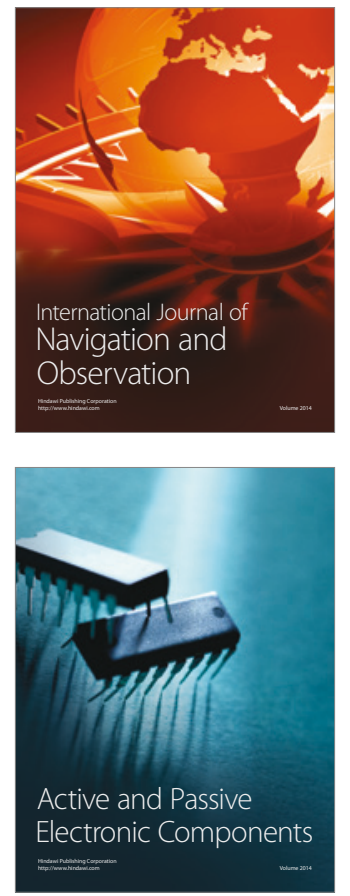
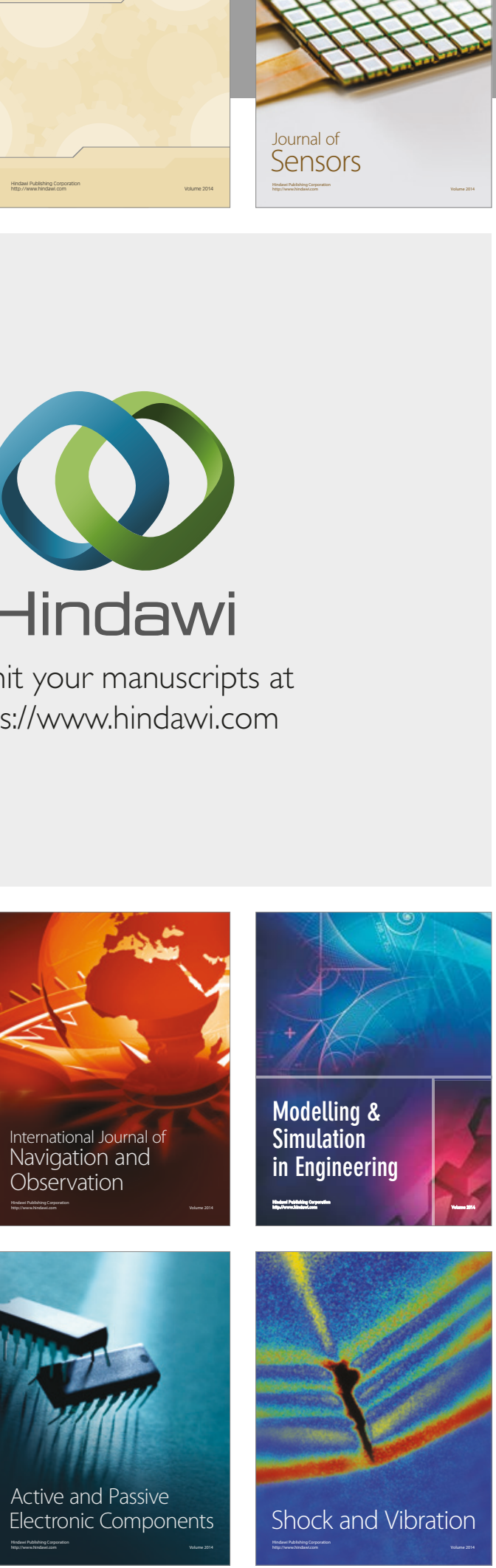
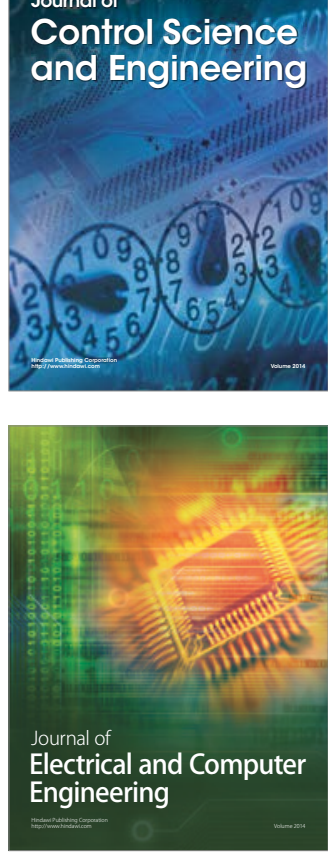

Distributed

Journal of

Control Science

and Engineering
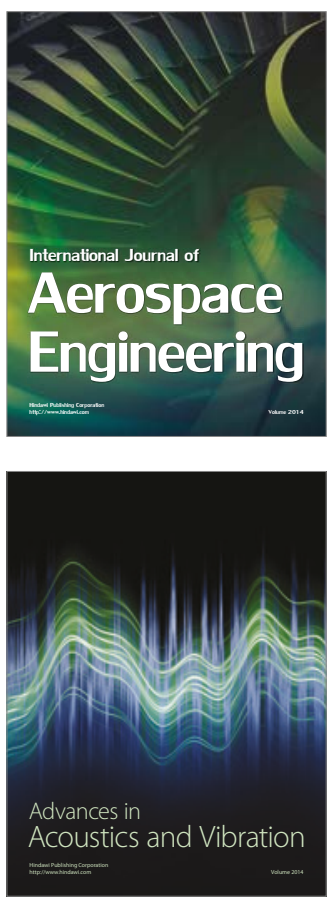

Sensor Networks 